\title{
Theoretical Model on Effects of the Flower Enterprises Networks to the Technical Innovative Performance
}

\author{
Dao-Wen REN ${ }^{1, a,{ }^{*}}$, Fei WEN ${ }^{2, b}$, Yan-Ji DUAN ${ }^{3, c}$ \\ ${ }^{1}$ Tianhe School, Guangdong Polytechnical Normal University, Guangzhou Guangdong, 510665, \\ China \\ ${ }^{2}$ South China Business College, Guangdong University of Foreign Studies, Guangzhou Guangdong, \\ 510545, China \\ ${ }^{3}$ School of business, Maryland University, Maryland State, 21201, USA

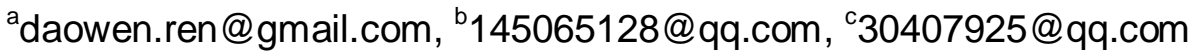 \\ ${ }^{*}$ Corresponding author
}

Keywords: Flower enterprises, Network structure, Entities' behaviors, Technical innovative performance.

\begin{abstract}
Combining existing related literatures, the paper analyzes and puts forward the concept models that the industry clusters' network affects enterprises' performance and raises some relevant research assumptions, by following the analytic logic of "structure-behaviors-performance", the theoretical frame "network structure-network body-network resource-technical innovation performance". It takes the lead to do the research in the unified theoretical model of the flower industry clusters' network structure, the bodies' behavior and the enterprises technical innovation performance. Systematic researches of the interaction effects of these variables are also preceded. The paper solves effectively that the previous research only used a specific variables to study the relationship of the specific variables and technical innovation performance. The innovative theoretical model provides a new tool of the scientific and explanatory power to illustrate the influence on the industrial cluster network to the enterprises technical innovation performance.
\end{abstract}

\section{Introduction}

The relevant study between the enterprises networks and the technical innovation performance has already become one of the crucial problems that the enterprises urgently wait to address and turned to be the hot spot in the current academia[1].Numerous academicians have already proved that the enterprises network exerts the direct positive influence over the technical innovation performance[2][3][4][5]. For those flower enterprises, there is no exception: the flower enterprises networks also affect their technical innovation performance[6][7][8]. Nevertheless, those academicians mentioned above mainly engrossed their attention on one aspect of the enterprises networks and rare is the situation that the network structure, the entity behavior and the technical innovation performance have been coordinated together under an integral theoretical framework. Therefore, this paper mainly analyzes whether and how the flower enterprises network structure and the entity behaviors affect the different enterprises' technical innovation performance. Finally, the result of the research can not only enrich the theory of the network and of the technical innovation, but also provide a valuable reference to the local government and the flower enterprises in manufacturing and establishing the relevant measures and the strategies to hoist the innovation performance.

The network consists of the behavioral entity, the happened activities and the resource [9]. In this article, we deem that the flower enterprises network is a kind of the network structure organization of competing and collaborating with both the upper and lower streams corporations, of correlating with non-corporation entities and of attaining and utilizing resource for the purpose of the improving innovation ability. The corporation entity exists in the enterprises' network, affected by the network structure while its resource utilization is determined by the network structure [10]. In the next step, 
the paper will analyze the relationship between the entity behavior, the network structure and the technical innovation performance.

\section{The relationship of the entity behavior and the technical innovation performance}

Gulati, Nohria \& Zaheer(2000) contended that the theory of the transitional social network only analyzes the relationship and the structure of the network while ignoring the attributes of the entities themselves[11]. And therefore, the emphasis of the future research should lie not only in the characteristics of the network structure, the quality of the network relationship, but also the attributes of the entities. Lavie(2008)also points out that the attributes of the network entities strongly affect the enterprises' compete actions[12]. The entities of the flower enterprises include the flower corporations, the local government, the colleges, the academic institutions, the medium agencies and the financial institutions, etc. The entities behaviors include the competitions and the collaborations among the corporation entities and the resource utilizations among non-corporation entities.

The competitions and the collaborations among the corporations and the technical innovation performance

The enterprises prove to be the most significant economic unit in the technical innovation investment and output within the flower enterprises' network. It is also the enterprise that maintains the conjunction of each entity within the network and that serve as the central part of the overall network. When the collaborations that based on the common manufacturing process happen among the flower enterprises, the producers, the suppliers and the salesmen are highly identical and coordinated, benefiting the knowledge communication within the network and stimulating the enormous innovations. When the enterprises correlate with their peer corporations, these correlation performed as the collaborations and the competitions and as the transmission and the pervasion in the horizontal level. The competitions and the collaborations together compel the enterprises to innovate continually and to establish their own innovative advantages, and thus to improve the innovation performance of those enterprises. Base on the analysis, we make the following hypothesis:

The hypothesis 1a: the competitions and the collaborations of the flower enterprises influence the enterprises technical innovation performance directly and remarkably.

\section{The resource utilization and the technical innovation performance}

The enterprises' networks reconstruct the resource of the corporation entities within the network. Although the bureaucracy organization process of the utilizing resource might seem to be non-exist, there is still spacious concentrating, upgrading and improving room for the enterprises in the network during the utilizing resource. What's more, it can also provide a flexible resource organizational advantage [13].This flexible resource organizational advantage can be mainly reflected when the corporations acquire and utilize resource. Within the network of the flower enterprises, the enterprises can attain and utilize the resource not by collaborating and competing among the corporations, but by working together with non-corporation entities. For instance, the subsidy, the policies and the information provided by the local government, the human resource, the knowledge and the technical resource accommodated by the colleges, the financial support given by the financial institutions and the information rendered by the agencies. Based on the analysis mentioned above, we make the following the hypothesis:

The hypothesis 1b: The resource utilization has a direct and remarkable effect to the enterprises' technical innovation performance.

\section{The relationship between the network structure and the technical innovation performance}

The characteristics of the network structure show and denote the interactive relationship among the network entities and exert a significant influence on the enterprises behaviors [14]. The network structure denotes the location of the network conjunction, the distribution of the connection of those 
conjunctions and the network density, etc [15].In this paper, we will lay our emphasis in studying the influence exerted by the network density, the network intensity and the network centricity to the technical innovation performance.

\section{The network density and the technical innovation performance}

The network density refers to the ratio of the enterprises number that real exists to the enterprises number that might can be contacted. This terminology is used to define the cohesion and the intimacy of each conjunction in the network. The network density can exert a special influence on the competitions and the collaborations of the corporation entities. Since the network density roots in the majority members of the enterprise entities within the network, it is much easier for them to share a common behavior anticipation and to form a coordinated guideline that is beneficial to the work divide within the network, and thus quicken the spread and share of the resource and improve the decision-making process as well as the behavior efficiency. The higher the network density is, the wider the connection among the enterprises entities, and the higher possibility that the technical innovation performance can be hoisted[16]. Within the flower enterprises network, the connection of the numerous entities can generate in a highly dense network. The dense network enlarge the flow of the resource within the network and meanwhile, the scope of acquiring and utilizing resource also expands as the entities connections increase. Resource acquiring and utilizing can be carried out among the multiple entities. Compare with the scanty network density, the dense network can help to achieve a higher resource obtain as well as the utilize efficiency. Zhoutao(2004)pointed out that the spreading scope and the range are much wider within the dense network, which means that the dense entities connections within the network represent a much more wide-spread ingest of the resource, distribution scope and speed of the resource utilization efficiency[17]. Based on the literature research and the analysis mentioned above, we put forward the following hypothesis:

The hypothesis 2a: The competitions and the collaborations of the flower enterprises serve as the mediate function in the network density impact to the technical innovation performance.

The hypothesis $2 \mathrm{~b}$ : The resource utilization serves as the mediate function in the network density impact to the technical innovation performance.

\section{The network intensity and the technical innovation performance}

The network intensity is defined as the time or the frequency that the entities in the network contact with other entities within a unit time[18]. In the theory of Granovette, the network intensity decides the quality of the information that the entities can obtain and the possibility of successfully achieving the individual goal. Within the enterprises network, the corporations establish a high frequency network connection with the suppliers and the clients, improving the stability of the network and suppressing the behaviors of the opportunism. Since the strong connection is somehow including the collaboration, the faith and the stability, the resource transfer can be fastened among the corporations. However, this punchy connection might oftentimes decline the diversity of the network resource. If the enterprises entities can maintain a high frequency interaction or transaction relation with the external organization, it is beneficial for them to ingest and make use of the knowledge, increase the technologies distinction and thus assist to create new products [19]. High network intensity can increase the inflow speed of the resource during their formal or informal associations of the flower enterprises, and therefore provide and allocate timely the resource, such as the human labors, the material, the capital, the technologies and the information, helping to boost the technical innovation performance. One of the requirements of improving technical innovation performance proves to be the intake and the utilization of the resource from the network since the flower enterprises are mainly medium-small sized enterprises that have a relatively weak endowment. Based on the literature research and the analysis mentioned above, we put forward the following hypothesis:

The hypothesis 3a: the competition and the collaboration of the flower enterprises serve as the mediate function in the network intensity impact to the technical innovation performance. 
The hypothesis $3 \mathrm{~b}$ : the resource utilization of the flower enterprises serves as the mediate function in the network intensity impact to the technical innovation performance.

\section{The network centrality and technical innovation performance}

The network centrality refers to the degree of the centrality of the entity within the overall network, representing the degree of the concentration of the power or the degree of the concentration. The entities be in the central of the network can procure and handle more resource since they can establish a direct connection with the enormous corporations entities, and thus making use of the resource at a shorter distance as well as an earlier time while decreasing the search cost[20].Meanwhile, the flower enterprises that lie in the central place within the network enjoy a higher degree of openness, which is beneficial to the expansion of the knowledge, and thus prove to be more competent than those less central enterprises. The centrality reflects the enterprises' ability to absorb and acquire the resource [21]. The enterprises that be in the central place can become the center of the network resource and serve as a pivot during the resource acquiring and the utilizing process, and thus be beneficial to hasten the corporations' decision process and to distribute the resource unanimously. It is much easier for the enterprises that placed in the network center to gain the strategic resource they need. In the meantime, it is also advantageous to promote the information variety and the resource diversity, which is critical for developing the new products and for generating the novelty ideas, and hence promoting the increase of the technical innovation performance. Based on the literature research and the analysis mentioned above, we put forward the following hypothesis:

The hypothesis 4a: the competitions and the collaborations of the flower enterprises serve as the mediate function in the network centrality impact to the technical innovation performance.

The hypothesis $4 \mathrm{~b}$ : the resource utilization serves as the mediate function in the network centrality impact to the technical innovation performance.

\section{The theoretical model and the hypothesis}

Based on the analysis mentioned above, we can get the following theoretical model and the hypothesis, shown in the figure one and in the timetable one.

\section{The theoretical model}

Around above the research problems, the paper gradually structures the theory model of the effect on the flower industrial cluster network to the technical innovation performance. The theory model illustrates the relation among the network structure, the enterprises bodies, the network resources and the technical innovation performance.

The flower industry clusters' network structure, the bodies' behavior and the enterprises technical innovation performance are integrated within the unified theoretical framework, which involves in the significant variables including the network structure, the enterprises bodies, the network resources and the technical innovation performance. The network structure is the explanatory variable, the enterprises bodies and the network resources are the intermediate variables, the technical innovation performance is the explained variable. The network structure is divided into three variables of the network density, the network intensity and the network centrality. The enterprises bodies are divided into three variables of the cooperation with the upstream firms, the cooperation with the downstream firms and the competition with the peers. The network resources are classified into four variables of the human resources, the technology, the fund and the information. The technical innovation performance are classified into six variables of the patent numbers that the per person have in the enterprises, the proportion of output value of the new product taking up total sales in the enterprises, the new product quantity, the speed of the new product development, the degree that the enterprises grasp the market or meet the customers, the new technology and the new methods that the enterprises import to improve the technological process. 


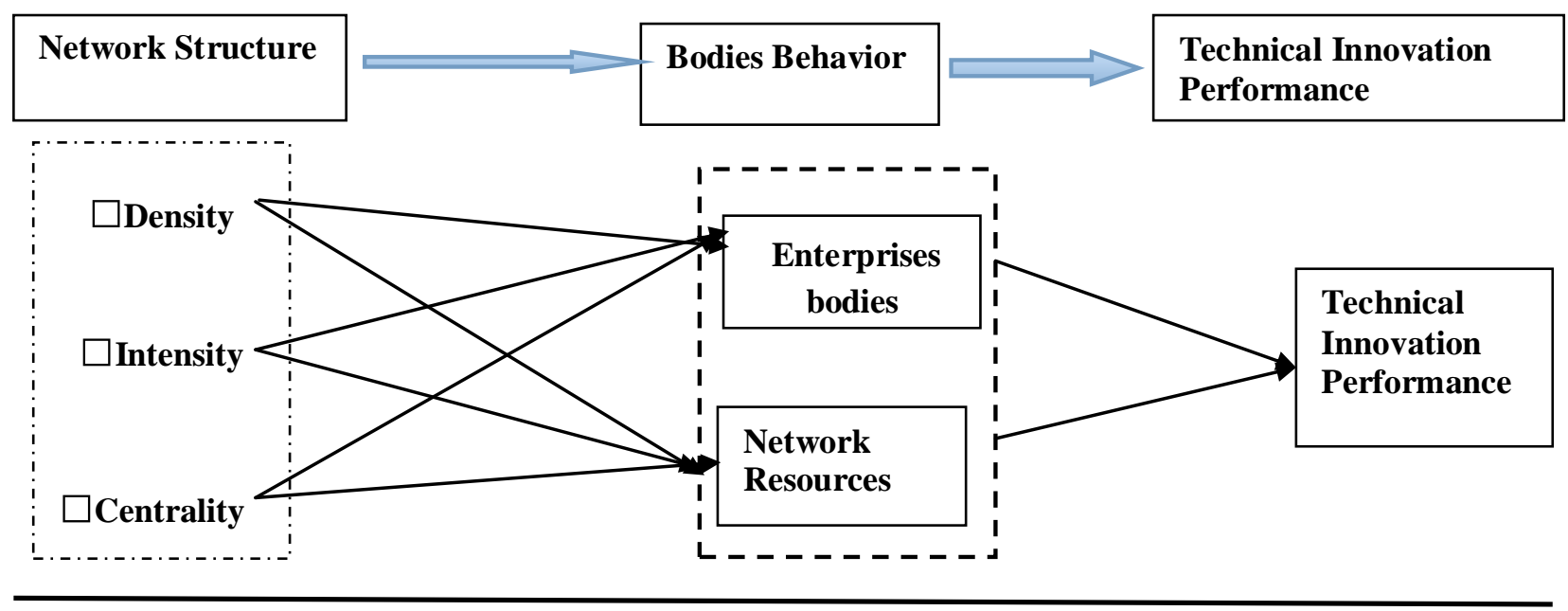

Fig 1 The theory model of effect on the flower industrial cluster network to technical innovation performance

\section{The hypothesis}

According to above the analysis, the hypotheses are made up three influences of the network structure, the enterprises bodies and the network resources to the technical innovation performance, shown in the timetable one.

Table 1 Research hypothesis of effect on the clusters'network to the enterprises' performance

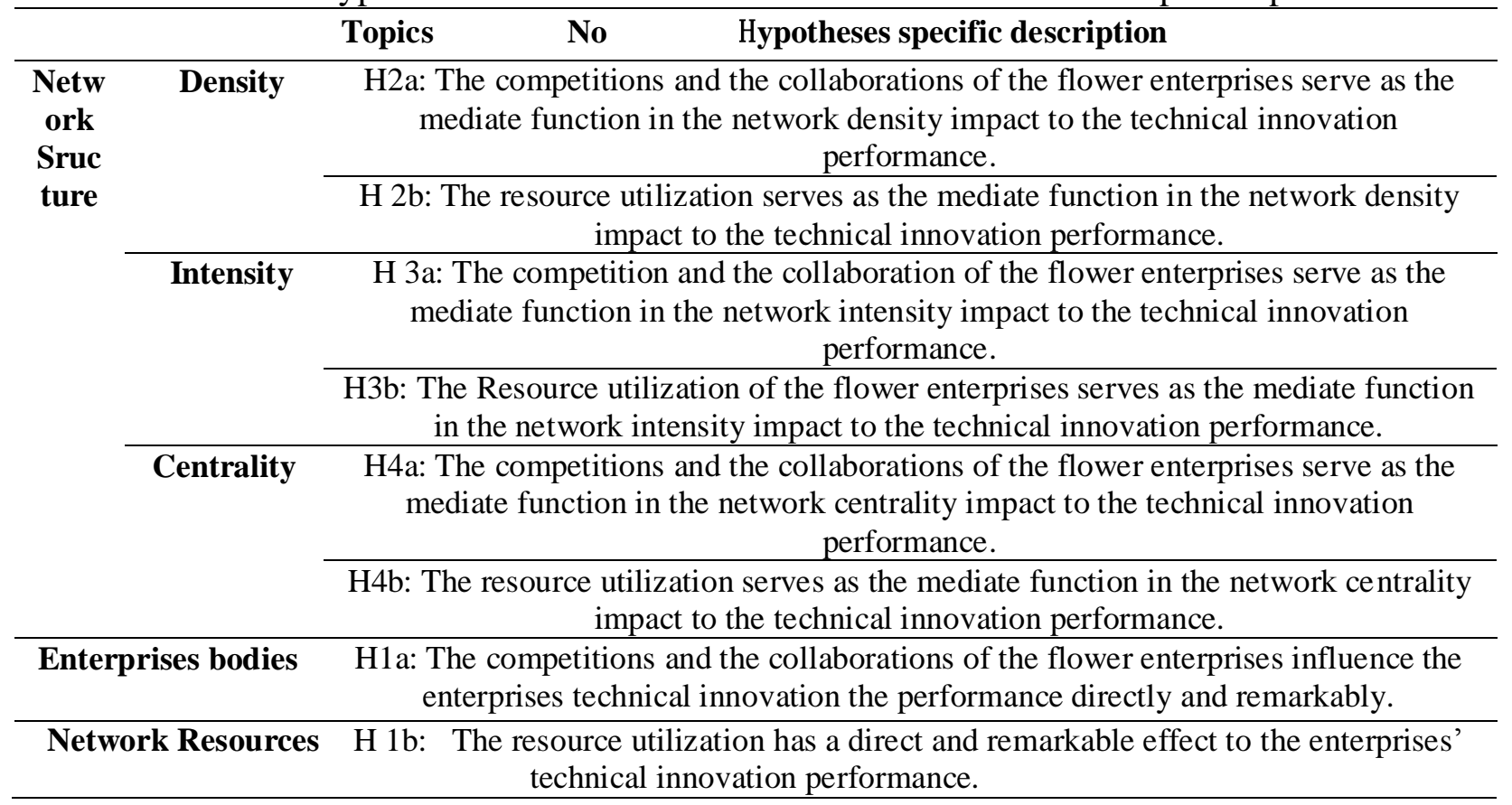

\section{Acknowledgements}

This research was financially supported by the Social Science Foundation of the China Education Department (11YJA630089).

\section{References}

[1] Xiaobo $\mathrm{Wu}, \mathrm{Wu}$ Dong. The system evolution of the innovative chain and its policy implication, J.Study on dialectics of nature. 24 (2008)58-62.

[2] Gargiulo, M.\&Benassi, M. Trapped in your own net? The network cohesion, The structural holes, and the adaptation of the social capital, J. Organization Science. 11(2000)183-196. 
[3]Meagher, K.\&Rogers, M. The network density and the R\&D spillovers, J.Journal of Economic Behavior\&Organization, 53(2004)237-260.

[4]Lazer, D.\&Friedman, A.The Network Structure of the Exploration and the Exploitation,J. Administrative Science Quarterly, 52(2007) 667-694.

[5]Xuefeng Liu. Relationship research on the network embedability, the differentiation strategy and the enterprises performance,D.PH D Thesis. Zhejiang University.2007.

[6]Chaminade C., Jan Vang. Upgrading in Asian Clusters: Rethinking the Importance of the Interactive Learning ,J.Science Technology \&Society,13(2008)61-94.

[7]Cortright Joseph,Provo John. Metropolitan Portland's Nursery Industry Cluster,R. Regional Connections(2000).

[8]Kusi Hornberger, Nick Ndiritu. Lalo Ponce-Brito, Melesse Tashu, Tijan Watt. Kenya's Cut-flower Cluster(2007).

[9] Hakansson, H. The Industrial technical development: A network approach,M.London: Croom Helm, (1987).

[10]Yongan Zhang, Chenguang Li. Influence research on the innovative network structure to the innovative resource usage ratio,J.Science and scientifical technical management, 1 (2010)81-89.

[11]Gulati, Nohria \& Zaheer:Strategic networks, J.Strategic Management Journal, 21(2000)203-215.

[12]Lavie, D. Network resources: Toward a new Social network perspective [J].Academy of Management Review, 33(2008)546-550.

[13]Cai Ling, jiebin Wu.The competition advantage of the enterprises cluster: the structural integration of the resource,J.Chinese Industry economics. 7 (2007).

[14]Jiebin Wu. Study on the Industry cluster competitive advantage based on the enterprises' network structure and the dynamic ability,D.PH D Thesis. Zhejiang University(2006).

[15]Soh, Pek-Hooi \& Roberts, Edward B. The Networks of the innovators: a longitudinal perspective. Research Policy,32(2003)1569-1588.

[16]Hongbin Du, Zhengbin Wang.The network structure,absorbing the capability and the enterprises' technical innovative performance,J.Chinese scientifical and technical forum,5 (2010) 25-30.

[17]Zhou Tao,Pinqun Jiang,Binghong Wang.Propagation behavior on the complex network. The complex network-system structure collected works,3 (2004).

[18]Granovetter,M.S.The strength of the weak ties, J.American Journal of Sociology,78 (1973)1360-1389.

[19]Gongqi Si. Relationship of the social capital and the small enterprises' growth,J.Science and scientifical technical management, 29(2008).

[20]Batjargal, B.\&Liu, M. The Social capital and the entrepreneurial performance in Russia:a longitudinal study,J. Organization Study, 24(2003)535-556.

[21]Hoang Ha, Antoncic Bostjan. The Network based the research in the entrepreneurship: A critical review,J. Journal of Management, 18(2003) 165-187. 